Article

\title{
Swelling Properties of Hydrogels Containing Phenylboronic Acids
}

\author{
Arum Kim ${ }^{1, \dagger}$, Siddharthya K. Mujumdar ${ }^{1, \dagger}$ and Ronald A. Siegel ${ }^{1,2, *}$ \\ 1 Department of Biomedical Engineering, University of Minnesota, Minneapolis, MN 55455, USA; \\ E-Mails: akim0105@gmail.com (A.K.); siddharthya.mujumdar@novartis.com (S.K.M.) \\ 2 Department of Pharmaceutics, University of Minnesota, Minneapolis, MN 55455, USA \\ $\dagger$ These authors contributed equally to this work. \\ * Author to whom correspondence should be addressed; E-Mail: siege017@umn.edu; \\ Tel.: +1-612-624-6164; Fax: +1-612-626-2125.
}

Received: 15 October 2013; in revised form: 19 November 2013 / Accepted: 12 December 2013 /

Published: 30 December 2013

\begin{abstract}
Phenylboronic acids are a class of compounds that bind glucose and other sugars. When polymerized into hydrogels, they provide a convenient nonenzymatic means for sensing glucose concentration, provided competing sugars are present at negligible concentrations. In this paper we provide a comprehensive study of swelling of hydrogels containing methacrylamidophenylboronic acid (MPBA), as a function of $\mathrm{pH}$ and concentration of either glucose or fructose. In one set of hydrogels, MPBA is substituted at $20 \mathrm{~mol} . \%$ in a polyacrylamide hydrogel [p(MPBA-co-AAm)], while in a second set of hydrogels, $20 \mathrm{~mol} \cdot \%$ MPBA is supplemented with $20 \mathrm{~mol} \% \%$ of $\mathrm{N}$-3-(dimethylaminopropyl methacrylamide) [p(MPBA-co-DMP-co-AAm)]. Swelling curves are markedly different for fructose and glucose, and for the two sets of hydrogels. While fructose alters swelling by binding and contributing to the ionization of MPBA, glucose does the same, but it also can form crosslinking bridges between separate chains, leading to hydrogel shrinkage. While the [p(MPBA-co-AAm)] hydrogels behaved as polyacids, swelling monotonically with increasing $\mathrm{pH}$, the [p(MPBA-co-DMP-co-AAm)] hydrogels exhibited polyampholyte behavior, with swelling minima at intermediate $\mathrm{pH}$ values.
\end{abstract}

Keywords: hydrogels; glucose sensing; polyelectrolyte; polyampholyte; Donnan osmotic pressure; phenylboronic acid 


\section{Introduction}

Diabetes mellitus is a condition in which control of blood sugar is compromised, either because the pancreas does not produce and secrete insulin properly (Type 1), or because the body's cells have decreased capacity to utilize the insulin that is secreted (Type 2). According to statistics released by the American Diabetes Association in 2011, 25.8 million children and adults in the United States (8.3\% of the population) have diabetes [1]. Type 2 diabetes is more prevalent, affecting $90 \%$ to $95 \%$ of the US diabetic population. Similar trends are observed worldwide, especially in developing countries where diet and lifestyle are changing. Without proper treatment, diabetes can cause many complications such as heart disease, stroke, high blood pressure, blindness, kidney disease, and neuropathy [2].

For long term management of diabetes, accurate measurements of blood glucose levels are essential. Blood glucose levels in diabetics exhibit large swings throughout the day. However, current widely used devices, such as test strips and glucose meters, give only discrete time information about blood glucose level, possibly missing fluctuations involving sudden increase or decrease in glucose level $[3,4]$. Recently commercialized enzyme based sensors such as Medtronic CGMS ${ }^{\circledR}$ Gold $^{\mathrm{TM}}$ and Dexcom $^{\circledR}$ G4 Platinum can offer continuous information, but they can be problematic due to instability of the enzyme, fouling under physiological conditions, and inflammation and infection that result from breaching the skin with a needle electrode [5]. While improved enzyme based sensors that minimize some of these issues are under development [6], parallel investigation of nonenzymatic sensing modalities is of continued interest.

One class of possible nonenzymatic glucose sensors involves derivatives of phenylboronic acid (PBA). When properly configured, PBA binds to glucose, other sugar molecules, and other polyols containing cis-diols. Binding can be observed by fluorescence change or by changes in swelling state of a hydrogel in which PBA moieties is incorporated. Several devices have been proposed based on the swelling/shrinking effect, including hydrogels containing colloidal crystal arrays that change color upon swelling and shrinking due to changes in Bragg spacing [7-11], holograms incorporated into hydrogels that also change color [12,13], hydrogel-coated tips of optical fibers that operate as Fabry-Perot interferometers [14,15], and confined, nearly isochoric hydrogels that exert swelling pressure on microfabricated confining structures, with pressure detected either piezoresistively [16-18] or electromagnetically $[19,20]$.

Figure 1 shows the simplest mechanism by which PBA responds to sugar molecules [21-24]. Under alkaline conditions boron, a Lewis acid with unfilled $\mathrm{sp}^{2}$ orbitals, becomes negatively charged by reaction with hydroxide ion, a Lewis base. Sugar molecules with planar cis-diols condense with the negatively charged tetrahedral boronate complex and stabilize the charged form. (Sugar condensation with the uncharged trigonal acid form of PBA also can occur, but much less frequently [8,25,26].) When attached to a hydrogel, these negatively charged $\mathrm{PBA}^{-}$groups engender an osmotic swelling force causing the hydrogel to expand. According to Figure 1, swelling will be facilitated by either high $\mathrm{pH}$ or high sugar concentration, or both. 
Figure 1. Complex between phenylboronic acid (PBA) moiety and a sugar molecule in aqueous solution $[8,25,26]$.
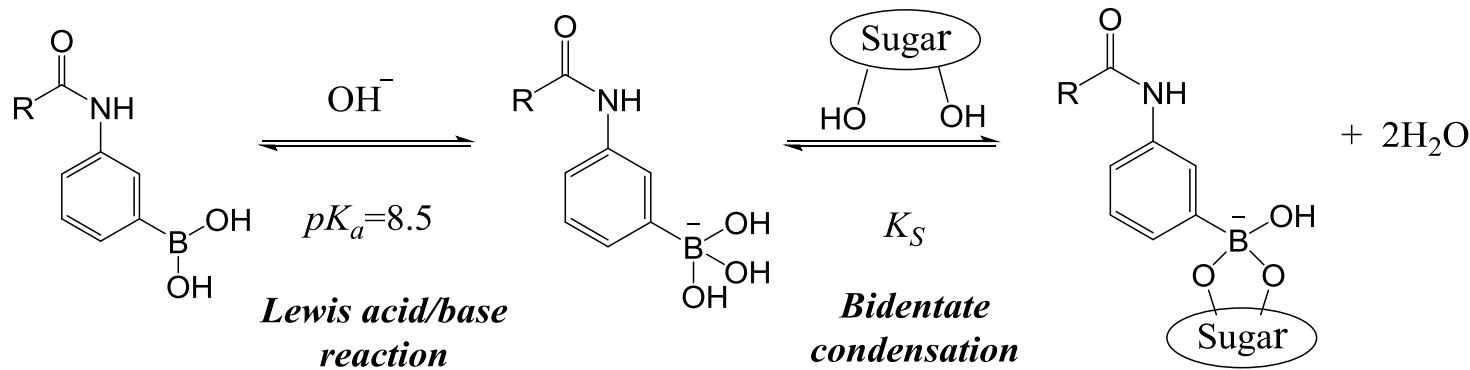

An important characteristic of any sensor, beyond its sensitivity to the target analyte, is its specificity. As already indicated, PBA binds other sugars besides glucose. The importance of this effect depends on the relative affinity of the PBA for glucose compared to that for each potential interfering species, and their respective abundances. Interestingly, however, there is one way in which glucose is distinct from other common sugars. Two sets of planar diols in a single glucose molecule can complex with PBA moieties on separate polymer chains, forming a bisbidentate bridge [8,12,27-29], as shown in Figure 2. By forming such (reversible) bridges, the hydrogel becomes effectively more crosslinked, causing shrinkage. Most other sugars such as fructose can bind to only one PBA moiety.

Figure 2. Proposed structures for glucose-PBA bridges between separate chains [8,12,27-29]. Glucose may be in its pyranose (left) or furanose (right) form. Adapted with permission from [12].
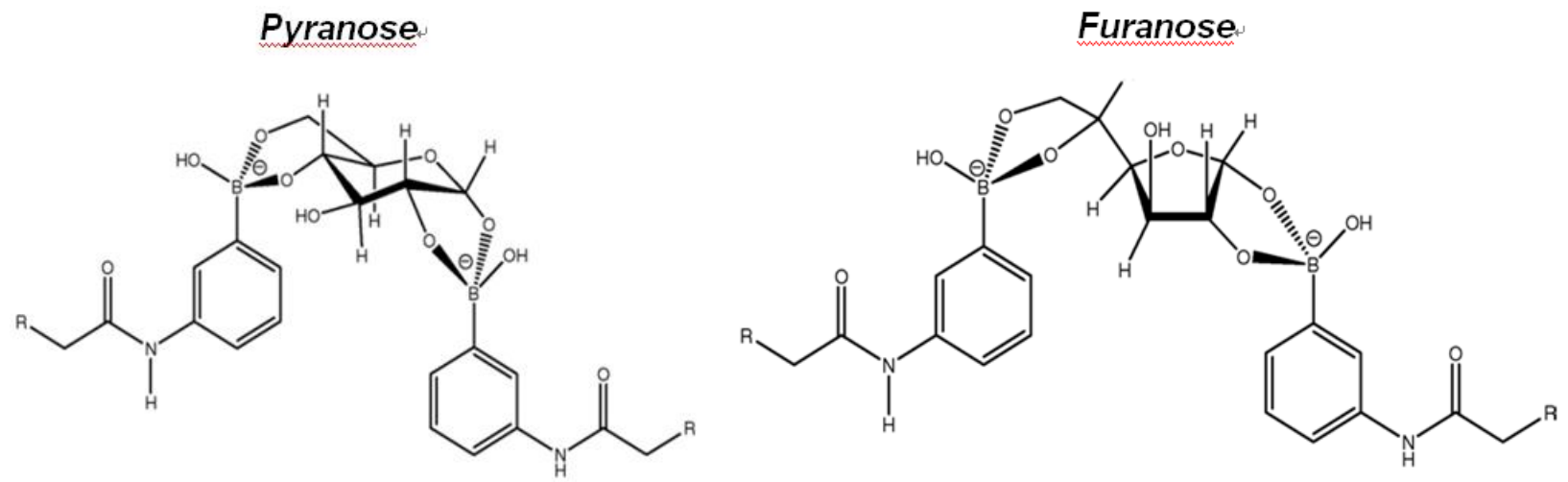

Figures 1 and 2 feature PBA linked through an amide bridge to a general group R. For the simplest hydrogels, $\mathrm{R}$ is an $\alpha$-methylated vinyl group, and the resulting monomer is 3 -methacrylamidophenylboronic acid (MPBA), which is polymerized into the hydrogel. The pKa for polymerized MPBA is approximately 8.5 [8]. The crosslink/shrink mechanism for glucose occurs in alkaline media above this $\mathrm{pKa}$, so it is not directly relevant to glucose sensing in vivo. Two strategies have been considered to lower the pKa of PBA. One is to introduce electron withdrawing groups such as nitro-, sulfonyl- and carboxyl groups on the benzene ring [10,23]. The other strategy is to place amine groups near the PBA moiety. In the latter case, an amine comonomer is copolymerized with the PBA-containing monomer, leading to a random copolymer structure [30-32]. The lone pair on the nitrogen in this neighboring amine group acts similarly to the hydroxide ion in the Lewis acid/base reaction described previously, 
associating with the unfilled $\mathrm{sp}^{2}$ orbital on the boron and lowering $\mathrm{pKa}$ to around $\mathrm{pH} 7$, as shown in Figure 3. This strategy improves glucose sensitivity at physiological $\mathrm{pH}$ and the specificity toward glucose molecules against other sugar molecules.

Figure 3. Interaction (dative bond) between PBA moiety and amine group in N,N'-3dimethylamino-propylmethacrylamide (DMP) near $\mathrm{pH} 7$.

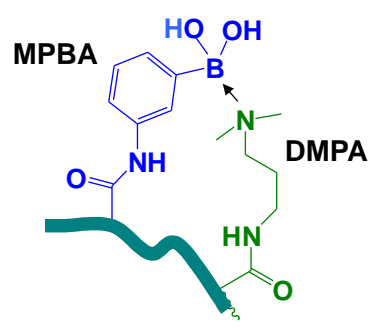

In this paper we present a study of the joint effects of $\mathrm{pH}$ and sugar concentration on free hydrogel swelling, for copolymers containing acrylamide (AAm), MPBA, and the crosslinker methylenebisacrylamide (Bis), and for terpolymers containing AAm, MPBA, N,N'-3-dimethylaminopropylmethacrylamide (DMP), and Bis. We have selected fructose and glucose for these studies, the results of which may guide those who wish to build sensors containing these hydrogels.

\section{Experimental Section}

\subsection{Synthesis of MPBA}

MPBA was synthesized from 3-aminophenylboronic acid hemisulfate $\left(\mathrm{PBA}^{+}\right)$by a procedure adapted with modification from [33]. Typically, $10 \mathrm{~g}$ of $\mathrm{PBA}^{+}$was dissolved in $160 \mathrm{~mL}$ distilled water placed in an ice bath $\left(0{ }^{\circ} \mathrm{C}\right)$. The $\mathrm{pH}$ of the solution was adjusted to 5.0 by adding $\mathrm{NaOH}$. Then, 1-ethyl-3-(3-dimethylaminopropyl) carbodiimide (EDAC) (12.34 g, $64.5 \mathrm{mmol}$ ) was added under continuous stirring in an argon atmosphere. The mixture was stirred for about 15 minutes. Meanwhile, $6.63 \mathrm{~g}(64.5 \mathrm{mmol})$ of methacrylic acid (MAA) was dissolved in $50 \mathrm{~mL}$ of distilled water and $\mathrm{pH}$ was again adjusted to 5.0. The resulting MAA solution was added dropwise to the $\mathrm{PBA}^{+} / \mathrm{EDAC}$ solution under argon. The entire mixture was purged with $\mathrm{Ar}$ at $0{ }^{\circ} \mathrm{C}$ for $90 \mathrm{~min}$. The mixture was then removed from the ice bath and stirred overnight at room temperature. Product was extracted with methyl $t$-butyl ether three times and roto-evaporated. The resulting white paste was dissolved in $380 \mathrm{~mL}$ distilled water at $70{ }^{\circ} \mathrm{C}$ with continuous stirring. Activated charcoal $(500 \mathrm{mg})$ was then added to the dissolved mixture, which was then stirred for $1 \mathrm{~min}$ at $70{ }^{\circ} \mathrm{C}$. The mixture was poured into a filter funnel to remove the charcoal. The final white crystal was obtained when the solution was left in the dark overnight under argon. The structure of MPBA was verified by ${ }^{1} \mathrm{H}-\mathrm{NMR}$.

\section{2.p(MPBA-co-AAm) Hydrogels}

Copolymer hydrogels containing MPBA and AAm were synthesized in an aqueous solution containing $20 \mathrm{~mol} \% \mathrm{MPBA}$ and $80 \mathrm{~mol} \% \mathrm{AAm}$ by redox copolymerization. The comonomers were dissolved in $1 \mathrm{~mL}$ of $1 \mathrm{~N} \mathrm{NaOH}, 80 \mu \mathrm{L}$ of $\mathrm{N}, \mathrm{N}, \mathrm{N}^{\prime}, \mathrm{N}^{\prime}$-tetramethyl ethylenediamine (TEMED: accelerator) and $100 \mu \mathrm{L}$ of $20 \mathrm{mg} / \mathrm{mL}$ Bis. Five parts of the resulting solution and one part of a 
$10 \mathrm{mg} / \mathrm{mL}$ solution of aqueous ammonium persulfate (APS: initiator) were then mixed. This pregel solution was loaded into silanized glass capillaries (I.D. $=1.172 \mathrm{~mm}$ ), and polymerized at $4{ }^{\circ} \mathrm{C}$ for $12 \mathrm{~h}$. Hydrogels were separated from the capillaries using acetone, cut into cylindrical segments $(\leq 1 \mathrm{~cm}$ long), and placed in distilled water at room temperature to extract unreacted materials. Before swelling experiments the hydrogels were equilibrated in $\mathrm{pH} 7.4$ phosphate buffer at room temperature.

\section{3.p(MPBA-co-DMP-co-AAm) Hydrogels}

Terpolymer hydrogels based on AAm, MPBA, and DMP were synthesized in dimethyl sulfoxide (DMSO). The composition was $60 \mathrm{~mol} \% \%$ AAm-20 mol.\% MPBA-20 mol.\% DMP. The monomers (total $5 \mathrm{mmol}$ ) along with $4.24 \mathrm{mg}$ 2,2'-azoisobutyronitrile (AIBN: initiator) and $100 \mu \mathrm{L}$ Bis $(20 \mathrm{mg} / \mathrm{mL}$ in DMSO) were dissolved in $1 \mathrm{~mL}$ of DMSO. Polymerization was carried out at $60{ }^{\circ} \mathrm{C}$ in silanized glass capillaries for $4 \mathrm{~h}$. Cylindrical hydrogels were prepared for swelling studies in the same way as with the AAm-co-MPBA hydrogels.

\subsection{Swelling Studies}

Equilibrium swelling studies were carried out at room temperature. The hydrogels were immersed in buffered $10 \mathrm{~mL}$ aqueous solutions, with various glucose or fructose concentrations $(0,0.5,2,7$ and $20 \mathrm{mM}$ ) at different $\mathrm{pH}$ values, spanning a range from $\mathrm{pH} 4$ to $\mathrm{pH} 10$. Swelling media consisted of $0.01 \mathrm{M} \mathrm{pH}$ buffers, adjusted to $0.154 \mathrm{M}$ ionic strength with $\mathrm{NaCl}$. Buffers were: acetic acid $(\mathrm{pKa}=4.76)$ from $\mathrm{pH} 4$ to $\mathrm{pH} 5.5$, sodium phosphate monobasic $(\mathrm{pKa}=7.2)$ from $\mathrm{pH} 6$ to $\mathrm{pH} 8$, and ethanolamine $(\mathrm{pKa}=9.5)$ from $\mathrm{pH} 8.5$ to $\mathrm{pH} 10$. The swelling medium for each sample was replaced daily. At the end of the swelling period (2-3 days), the final $\mathrm{pH}$ of the swelling medium was determined for each condition, and equilibrium diameter changes of the hydrogel cylinders were measured under an optical microscope attached to a video monitor with movable crosshairs.

\section{Results and Discussion}

All results are reported as the ratio of hydrogel cylinder diameter at swelling equilibrium $(d)$ to the diameter at synthesis $\left(d_{0}\right)$, taken to be the inner diameter of the capillary, $1.172 \mathrm{~mm}$. Final $\mathrm{pH}$ values were typically very close to the original values, and equilibrium was typically achieved within one day.

\section{1.p(MPBA-co-AAm) Hydrogels}

Results of pH-dependent swelling studies of $\mathrm{p}$ (MPBA-co-AAm) copolymer hydrogels in the presence of different concentrations of fructose and glucose are shown in Figure 4a,b, respectively. In all cases, an increase of swelling with increasing $\mathrm{pH}$ is observed. This behavior is attributed primarily to increased Donnan osmotic pressure and change in hydrophilicity of the MPBA units due to ionization. With increasing $\mathrm{pH}$, more hydroxide ions are present, which convert the trigonal PBA moiety to the charged tetrahedral form. The latter is further stabilized by condensation with a sugar molecule, as discussed above. The charged $\mathrm{PBA}^{-}$moiety draws in counterions from the surrounding solution, and the resulting excess of mobile ions inside the hydrogel generates osmotic influx of water, causing the 
hydrogel to swell. Also, the stabilized charge may alter the polarity and enhance the hydrophilicity of MPBA, promoting swelling.

For the experiment with fructose (Figure 4a), a simple acid shift is observed in the swelling curves with increasing fructose concentration. The acid shift corresponds to a change in apparent pKa of PBA due to stabilization of the charged form (Figure 1), which can be expressed [34] as

$$
p K_{a p p}=p K_{a}-\log \left(1+\frac{C_{s}}{K_{S}}\right)
$$

where $C_{S}$ is fructose concentrations and $K_{S}$ is the dissociation constant of fructose and ionized MPBA. The estimated $K_{s}$ value based on Equation (1) and the swelling curves in Figure 4a is $0.094 \mathrm{mM}$. This value is different from the literature value [34] for binding of fructose to free ionized PBA, $0.27 \mathrm{mM}$, but it is consistent with observations made previously with colloidal crystal-embedded hydrogels containing MPBA [8,9]. The slight increase in the plateau swelling at high $\mathrm{pH}$ values in the presence of fructose suggests that fructose-bound PBA is slightly more hydrophilic than bare ionized PBA.

Figure 4. Equilibrium swelling of methacrylamidophenylboronic acid (MPBA)-co-AAm hydrogels in buffer solution at different fructose $(\mathbf{a})$; or glucose $(\mathbf{b})$; concentrations $(n=2)$.

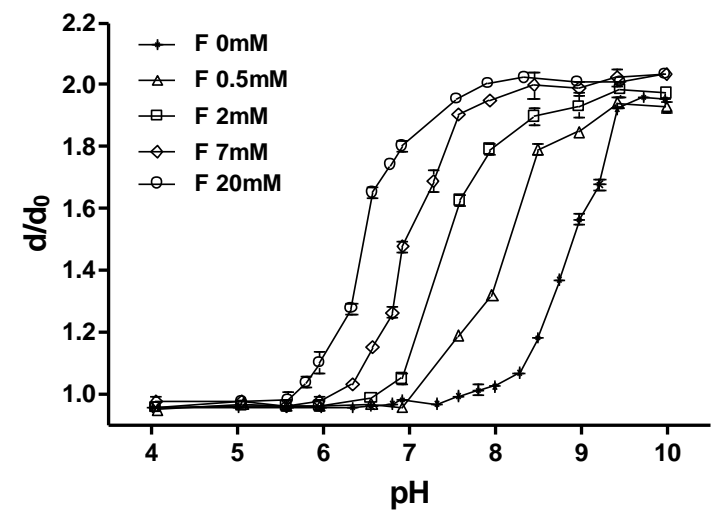

(a)

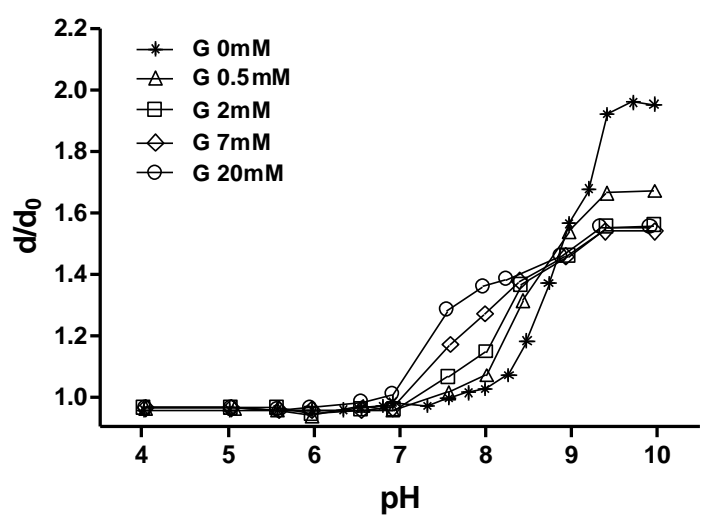

(b)

The response to glucose, detailed in Figure $4 \mathrm{~b}$, is more complicated. Below pH 8.8, glucose effects an acid shift in swelling as with fructose, but the degree of swelling of hydrogels in glucose solutions is much less than that in fructose solutions at the same $\mathrm{pH}$ and the same sugar concentration. This observation is explained by less avid binding of glucose to PBA, compared to fructose $[9,20]$. Moreover, while increasing fructose concentration always leads to an increase in swelling, increased glucose concentration leads to shrinkage of the hydrogel above $\mathrm{pH}$ 8.5. This is evident from the crossing over of swelling curves seen near $\mathrm{pH} 8.5$ in Figure $4 \mathrm{~b}$. Binding of glucose molecules to PBA moiety affects the swelling of hydrogels in two ways. First, binding of glucose increases the charge density of the hydrogel, increasing Donnan osmotic pressure and hydrophilicity, causing the hydrogel to swell, in the same way as with fructose. Second, formation of bisbidentate complexes or bridges, which occurs with glucose (see Figure 2) but not with fructose, increases the effective number of crosslinks in the hydrogel, causing it to shrink. Below pH 8.5, the first effect dominates, while the second effect makes a significant contribution above $\mathrm{pH}$ 8.5. This is the reason for the differing behaviors of the swelling between fructose and glucose in p(MPBA-co-AAm) hydrogels. 


\section{2. p(MPBA-co-DMP-co-AAm) Hydrogels}

Results of $\mathrm{pH}$-dependent swelling studies of $\mathrm{p}$ (MPBA-co-AAm-co-DMP) terpolymer hydrogels in the presence of different concentrations of fructose and glucose are presented in Figure 5a,b, respectively. Whereas swelling of the p(MPBA-co-AAm) copolymer hydrogels always increases with pH (Figure 4), the swelling curves for the terpolymer hydrogels exhibit definite nonmonotonic behavior, characteristic of polyampholyte hydrogels $[35,36]$, with maximum swelling at the extreme $\mathrm{pH}$ values and a swelling minimum near neutral $\mathrm{pH}$. Below $\mathrm{pH} 6$, addition of sugar scarcely affects hydrogel swelling. Above $\mathrm{pH}$ 6, however, addition of fructose (Figure 5a) increases swelling, and there is an acid shift in the swelling minimum. In contrast, addition of glucose (Figure 5b) causes shrinkage. For sugar free hydrogels, and with addition of fructose, swelling is higher in the alkaline extreme, while the opposite is the case when glucose is added.

Figure 5. Equilibrium swelling of DMP-co-MPBA-co-AAm hydrogels in buffer solution at different fructose $(\mathbf{a})$; or glucose $(\mathbf{b})$; concentrations $(n=2)$.

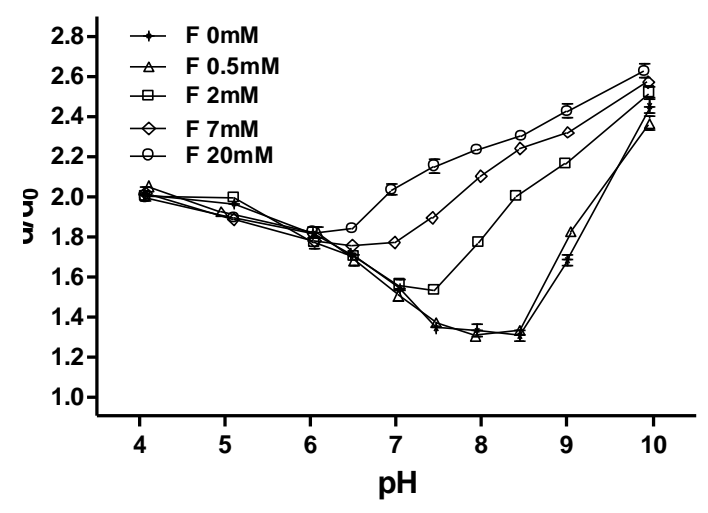

(a)

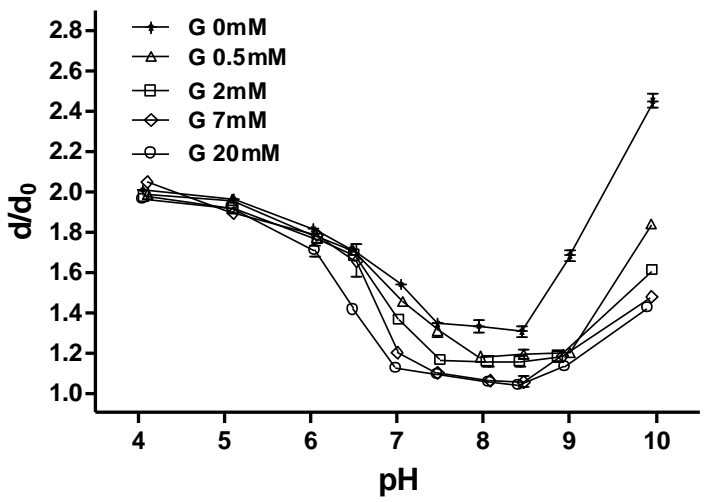

(b)

We can explain the different swelling behavior of the DMP containing hydrogels based on the change in the interaction between the PBA moiety and the neighboring amine group, as shown in Figure 6 [30]. We first consider the swelling behavior in the absence of sugar. At low $\mathrm{pH}$, where there are plenty of hydrogen ions available in solution, the nitrogen on the amine group is protonated (pKa 9), changing it to the positively charged state, while the PBA moiety remains uncharged. The net charge is therefore positive, leading to a Donnan osmotic pressure, and swelling of the hydrogel. At high $\mathrm{pH}$, the PBA moiety becomes negatively charged by Lewis acid/base reaction with hydroxide ion, while the amine group loses its charge, so the charge on the hydrogel becomes negative, again leading to Donnan osmotic pressure driven swelling. The asymmetry in swelling between high and low $\mathrm{pH}$ conditions might be due either to (a) a small excess of MPBA over DMP in the hydrogel, (b) greater hydrophilicity of the negatively charged MPBA compared to positively charged DMP, or (c) greater hydrophobicity of MPBA in its uncharged state compared to DMP. Such swelling asymmetries are common in other polyampholyte hydrogels $[35,36]$. 
Figure 6. Acid-base equilibrium of PBA moiety in DMP-co-MPBA-co-AAm hydrogel.

The B-N bond at intermediate $\mathrm{pH}$ is shown to be zwitterionic, though it may only be dipolar.

\section{Low pH}

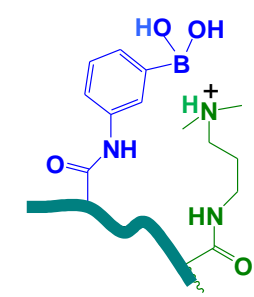

Intermediate $\mathrm{pH}$

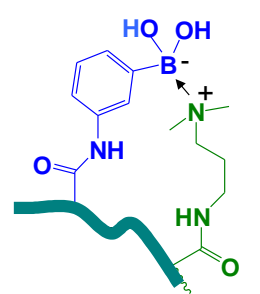

High pH

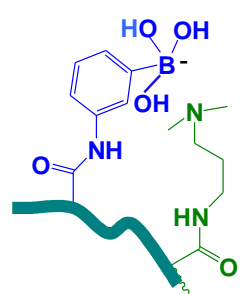

At intermediate $\mathrm{pH}$ values, two things can occur, both promoting shrinkage of the hydrogel. First, in this range the charges on PBA and DMP moieties may cancel each other out [37]. Second, the lone pair of electrons on the nitrogen in the tertiary amine group may associate with the electron deficient boron in the PBA moiety, forming a dative bond, which will have strong dipolar character and may even be zwitterionic. As the negative charge of boronate and the positive charge of nitrogen are canceled out, the overall charge in the system is neutral, so relative shrinkage compared to the extreme $\mathrm{pH}$ cases is expected. It is also well known that the presence of nearly equal amounts of positively and negatively charged groups, along with zwitterions, may promote extra shrinkage in hydrogels via the so-called polyampholyte effect [35,36], whereby equally matched fixed positive and negative charges attract each other, although this attraction is partially screened by mobile ions.

It may be noted that swelling of the terpolymer gels in the net neutral state is greater than for the copolymer hydrogels not containing DMP. This is somewhat puzzling, since DMP is expected to be more hydrophobic than AAm. However, direct quantitative comparison is probably not appropriate because the two types of hydrogels were synthesized in different solvents. To check this effect, limited swelling studies were carried out with p(MPBA-co-AAm) hydrogels synthesized in DMSO. It was found (data not shown) that the swelling baseline was higher, with $d / d_{0}$ close to 1.1 , than for the same hydrogels synthesized in water (Figure 4 ), where $d / d_{0}$ was slightly less than 1.0 at baseline.

We now turn to the effects of added sugar molecules. Note first that in the terpolymer hydrogels, PBA can undergo a Lewis acid/base reaction with either $\mathrm{OH}^{-}$in solution or with neighboring DMP groups. In either case the resulting tetrahedral $\mathrm{PBA}^{-}$moiety can complex with sugar molecules. It should be noted however that the MPBA-DMP complex does not carry a net charge, so binding of a sugar molecule to such a complex, while potentially stabilizing it, does not in itself promote charging of the network. On the other hand, binding of sugar to a lone $\mathrm{PBA}-\mathrm{OH}^{-}$complex will stabilize that latter's charge.

At any particular $\mathrm{pH}$, addition of fructose either causes swelling to occur or there is no significant effect. Roughly, the swelling curves in Figure 5a resemble a superposition of the fructose effect observed in Figure $4 \mathrm{a}$ for $\mathrm{p}$ (MPBA-co-AAm) hydrogels, with the polyampholyte behavior of the p(MPBA-coDMP-co-AAm) hydrogels observed with no sugar added. This resemblance is not quantitive, and there are some small but perhaps significant discrepancies. For example, the fructose effect is stronger at pH 10 in the terpolymer hydrogels than in the copolymer hydrogels, and the small effect of $20 \mathrm{mM}$ fructose on swelling of the copolymer hydrogel at $\mathrm{pH} 6$ is not seen in the terpolymer hydrogel. 
As noted before, these differences may be due to the different solvents used to prepare the copolymer and terpolymer hydrogels.

Conversely, the presence of glucose shrinks the terpolymer hydrogels at all $\mathrm{pH}$ values where it has an effect. In this case note that a glucose molecule can act as a crosslinker for Lewis base form $\mathrm{PBA}^{-}$ groups, regardless of whether this configuration arises from interaction of MPBA with $\mathrm{OH}^{-}$or DMP. At high $\mathrm{pH}$, it is the charged $\mathrm{MPBA}-\mathrm{OH}^{-}$groups that are crosslinked by glucose, while at intermediate $\mathrm{pH}$ values, it is the MPBA-DMP complexes that are involved. Below $\mathrm{pH}$ 6, glucose does not bind MPBA, so there is no crosslinking.

Thus far in this report, swelling response has been characterized in the presence of single sugars, without interference between them. Figure 7 demonstrates the effect of added fructose on glucose induced swelling and shrinking of similar hydrogels at $\mathrm{pH}$ 7.4. For convenience, swelling ratios are normalized to those observed in sugar free PBS at $\mathrm{pH} 7.4$ [38]. When fructose is presented at $100 \mu \mathrm{M}$, well above its mean physiological level [39], it has a minor but detectable effect on glucose response for $\mathrm{p}$ (MPBA-co-AAm) hydrogels, while for p(MPBA-co-DMP-co-AAm) hydrogels, no effect is observed. Beyond $100 \mu \mathrm{M}$, swelling increases with increasing addition of fructose at all glucose levels, for both hydrogels.

Figure 7. Effect of added fructose on glucose induced swelling (MPBA-co-AAm hydrogels: solid symbols) and shrinking (DMP-co-MPBA-co-AAm hydrogels: unfilled symbols) at $\mathrm{pH} 7$.

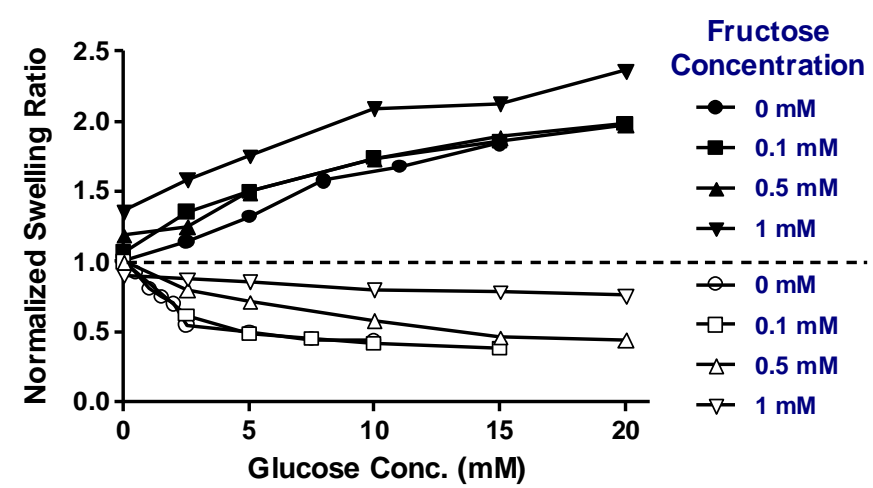

\section{Conclusions}

In this paper we have reported the results of swelling studies of $\mathrm{pH}$ - and sugar sensitive polyacid copolymer and polyampholyte terpolymer hydrogels at different $\mathrm{pH}$ values, in the presence or absence of sugar molecules. In the swelling studies of the polyacidic p(MPBA-co-AAm) hydrogel with no sugar added, a typical swelling transition was observed near the pKa for MPBA. The swelling curve exhibited an acid shift upon addition of fructose, which could be explained by stabilization of the charged form of MPBA upon complexation with fructose. Glucose acts on the hydrogel in two ways. First, it stabilizes charged MPBA, thus enhancing swelling, as does fructose, although with less avidity. Second, glucose can complex with two MPBA units simultaneously, leading to extra transient but active crosslinks, which cause the hydrogel to shrink. Whereas glucose promotes swelling below the pKa of MPBA, it leads to shrinkage above the pKa. The amine containing p(MPBA-co-AAm-co- 
DMP) hydrogels exhibit expected polyampholyte behavior. At $\mathrm{pH} 6$ and above, addition of fructose always leads to increased swelling, while glucose always causes shrinking. Importantly, the shrinking responses occur at physiological $\mathrm{pH}$, suggesting that the shrinking mechanism can be used in biomedical sensing and actuation. For the shrinking mode, fructose at physiological levels interferes negligibly with glucose response.

\section{Acknowledgments}

This work was supported in part by NIH Grants HD040366 and DK075739.

\section{Conflicts of Interest}

The authors declare no conflict of interest.

\section{References}

1. National Diabetes Fact Sheet, 2011. http://www.cdc.gov/diabetes/pubs/pdf/ndfs_2011.pdf (accessed on 26 December 2013).

2. The Diabetes Control and Complications Trial Research Group. The effect of intensive treatment of diabetes on the development and progression of long term complications in insulin-dependent diabetes mellitus. N. Engl. J. Med. 1993, 329, 977-986.

3. Gough, D.A.; Armour, J.C.; Baker, D.A. Advances and prospects in glucose assay technology. Diabetologia 1997, 40, S1025-1107.

4. Klonoff, D. Noninvasive blood glucose monitoring. Diabetes Care 1997, 20, 433-437.

5. Koschwanez, H.E.; Reichert, W.M. In vitro, in vivo and post explantation testing of glucosedetecting biosensors: Current methods and recommendations. Biomaterials 2007, 28, 3687-3703.

6. Gough, D.A.; Kumosa, L.S.; Routh, T.L.; Lin, J.T.; Lucisano, J.Y. Function of an implantanted tissue glucose sensor for more than 1 year in animals. Science Transl. Med. 2010, 2, 39-42.

7. Alexeev, V.; Das, S.; Finegold, D.; Asher, S. Photonic crystal glucose-sensing material for noninvasive monitoring of glucose in tear fluid. Clin. Chem. 2004, 50, 2353-2360.

8. Alexeev, V.L.; Sharma, A.C.; Goponenko, A.V.; Das, S.; Lebedev, I.K.; Wilcox, C.S.; Finegold, D.N.; Asher, S.A. High ionic strength glucose-sensing photonic crystal. Anal. Chem. 2003, 75, 2316-2323.

9. Asher, S.A.; Alexeev, V.L.; Goponenko, A.V.; Sharma, A.C.; Lednev, I.K.; Wilcox, C.S.; Finegold, D.N. Photonic crystal carbohydrate sensors: Low ionic strength sugar sensing. J. Am. Chem. Soc. 2003, 125, 3322-3329.

10. Muscatello, M.; Stunja, L.E.; Asher, S.A. Polymerized crystalline colloidal array sensing of high glucose concentration. Anal. Chem. 2009, 81, 4978-4986.

11. Lee, Y.-J.; Pruzinsky, S.A.; Braun, P.V. Glucose-sensitive inverse hydrogel opals: Analysis of optical diffraction response. Langmuir 2004, 20, 3096-3106.

12. Horgan, A.M.; Marshall, A.J.; Kew, S.J.; Dean, K.E.S.; Creasey, C.D.; Kabilan, S. Crosslinking of phenylboronic acid receptors as a means of glucose selective holographic detection. Biosens. Bioelectron. 2006, 21, 1838-1845. 
13. Kabilan, S.; Marshall, A.J.; Sartain, F.K.; Lee, M.-C.; Hussain, A.; Yang, X.; Blyth, J.; Karangu, N.; James, K.; Zeng, J.; Smith, D.; Domschke, A.; Lowe, C.R. Holographic glucose sensors. Biosens. Bioelectron. 2005, 20, 1602.

14. Tierney, S.; Falch, B.M.; Hjelme, D.R.; Stokke, B.T. Determination of glucose levels using a functionalized hydrogel-optical fiber biosensor: Toward continuous monitoring of blood glucose in vivo. Anal. Chem. 2009, 81, 3630-3636.

15. Tierney, S.; Volden, S.; Stokke, B.T. Glucose sensors based on a responsive gel incorporated as a fabry-perot cavity on a fiber-optic readout platform. Biosens. Bioelectron. 2009, 24, 2034-2039.

16. Lin, G.; Chang, S.F.; Hao, H.; Tathireddy, P.; Orthner, M.; Magda, J.J.; Solzbacher, F. Osmotic swelling pressure response of smart hydrogels suitable for chronically implantable glucose sensors. Sens. Act. B 2010, 144, 332-336.

17. Orthner, M.; Lin, G.; Avula, M.; Buetefisch, S.; Magda, J.J.; Rieth, L.W.; Solzbacher, F. Hydrogel based sensor arrays $(2 \times 2)$ with perforated piezoresistive diaphragms for metabolic monitoring (in vitro). Sens. Act. B. 2010, 145, 807-816.

18. Guenther, M.; Gerlach, G.; Wallmersperger, T. Piezoresistive biochemical sensors based on hydrogels. Microsyst. Technol. 2010, 16, 703-715.

19. Lei, M.; Baldi, A.; Nuxoll, E.; Siegel, R.A.; Ziaie, B. A hydrogel based implantable micromachined transponder for wireless glucose measurement. Diabet. Technol. Therap. 2006, 8 , 112-122.

20. Siegel, R.A.; Gu, Y.; Lei, M.; Baldi, A.; Nuxoll, E.; Ziaie, B. Hard and soft micro- and nanofabrication: An integrated approach to hydrogel based sensing and drug delivery. J. Control. Rel. 2010, 141, 303-313.

21. Kataoka, K.; Miyazaki, H.; Bunya, M.; Okano, T.; Sakurai, Y. Totally synthetic polymer gels responding to external glucose concentration: Their preparation and appliation to on-off regulation of insulin release. J. Am. Chem. Soc. 1998, 120, 12694-12695.

22. Matsumoto, A.; Yamamoto, K.; Yoshida, M.; Kataoka, K.; Aoyagi, T.; Miyahara, Y. A totally synthetic glucose responsive gel operating in physiological aqueous conditions. Chem. Commun. 2010, 46, 2203-2205.

23. Matsumoto, A.; Yoshida, R.; Kataoka, K. Glucose-responsive polymer gel bearing phenylborate derivative as a glucose-sensing moiety operating at physiological pH. Biomacromolecules 2004 , $5,1038-1045$.

24. Shiino, D.; Murata, Y.; Kataoka, K.; Koyama, Y.; Yokoyama, M.; Okano, T.; Sakurai, Y. Preparation and characterization of a glucose-responsive insulin-releasing polymer device. Biomaterials. 1994, 15, 121-128.

25. Springsteen, G.; Wang, B. A detailed examination of boronic acid-diol complexation. Tetrahedron 2002, 58, 5291-5300.

26. Yan, J.; Springsteen, G.; Deeter, S.; Wang, B. The relationship among pKa, pH, and binding constants in the interactions between boronic acids and diols-It is not as simple as it appears. Tetrahedron 2004, 60, 11205-11209.

27. Siegel, R.A.; Gu, Y.; Baldi, A.; Ziaie, B. Novel swelling/shrinking behaviors of glucose-binding hydrogels and their potential use in a microfluidic delivery system. Macromol. Symp. 2004, 208, 249-256. 
28. Pan, X.; Yang, X.; Lowe, C.R. Evidence for a cross-linked mechanism underlying glucoseinduced contraction of phenylboronate hydrogel. J. Mol. Recognit. 2008, 21, 205-209.

29. Ancla, C.; Lapeyre, V.; Gosse, I.; Catargi, B.; Ravaine, V. Designed glucose-responsive microgels with selective shrinking behavior. Langmuir 2011, 27, 12693-12701.

30. Shiino, D.; Kubo, A.; Murata, Y.; Koyama, Y.; Kataoka, K.; Kikuchi, A.; Sakurai, Y.; Okano, T. Amine effect on phenylboronic acid complex with glucose under physiological $\mathrm{pH}$ in aqueous solution. J. Biomater. Sci. Polymer Ed. 1996, 7, 697-705.

31. Kitano, S.; Hisamitsu, I.; Koyama, Y.; Kataoka, K.; Okano, T.; Sakurai, Y. Effect of the incorporation of amino groups in a glucose-responsive polymer complex having phenylboronic acid moieties. Polym. Adv. Technol. 1991, 2, 261-264.

32. Hisamitsu, I.; Kataoka, K.; Okano, T.; Sakurai, Y. Glucose-responsive gel from phenylborate polymer and poly(vinyl alcohol): Prompt response at physiological $\mathrm{pH}$ through the interaction of borate with amino group in the gel. Pharm. Res. 1997, 14, 289-293.

33. Shiino, D.; Koyama, Y.; Kataoka, K.; Yokoyama, M.; Okano, T.; Sakurai, Y. Design of glucose responsive, insulin releasing device using polymers containing boronic acid groups. J. Artif. Org. 1992, 21, 1196-1198.

34. Gu, Y. Swelling Properties of Phenylboronic Acid-Containing Hydrogels and Their Applications in Microfluidic Drug Delivery Devices. Ph.D. Thesis, University of Minnesota, Minneapolis, MN, USA, 2003.

35. Baker, J.; Stephens, D.; Blanch, H.; Prausnitz, J. Swelling equilibra for acrylamide based polyampholyte hydrogels. Macromolecules 1992, 25, 1955-1958.

36. English, A.; Mafe, S.; Mazanares, J.A.; Yu, X.; Grosberg, A.Y.; Tanaka, T. Equilibrium swelling properties of polyampholytic hydrogels. J. Chem. Phys. 1996, 104, 8713-8720.

37. Hoare, T.; Pelton, R. Charge-switching, amphoteric glucose-responsive microgels with physiological swelling activity. Biomacromolecules 2008, 9, 733-740.

38. Mujumdar, S.K. Stimuli Sensitive Hydrogels for Controlled Drug Delivery and Sensing Applications; University of Minnesota: Minneapolis, MN, USA, 2007.

39. Kawasaki, T.; Ahanuma, H.; Yamanouchi, T. Increased fructose concentrations in blood and urine in patients with diabetes. Diabetes Care 2002, 25, 353-357.

(C) 2013 by the authors; licensee MDPI, Basel, Switzerland. This article is an open access article distributed under the terms and conditions of the Creative Commons Attribution license (http://creativecommons.org/licenses/by/3.0/). 